\title{
Games, from Engaging to Understanding: a Perspective from a Museum of Computing Machinery
}

\author{
Giovanni A. Cignoni, Leonora Cappellini, Tommaso Mongelli \\ Fondazione Galileo Galilei, Museo degli Strumenti per il Calcolo \\ Lungarno Pacinotti 43-44 c/o Università di Pisa - 56126 Pisa \\ giovanni@di.unipi.it, leonora.cappellini@gmail.com,mog_tom@yahoo.it
}

\begin{abstract}
Science museums have a natural role in the building of public understanding of science. For some time now, museums are particularly focused on engaging the public: it is a necessary condition to raise interest and cause active responses from the public. In this context, gamification, that is the usage of game dynamics to drive participation, is a way to engage the public.

The paper presents the experiences of the Museum of Computing Machinery of the University of Pisa in the adoption of gaming approaches for attracting and involving its public. Being a museum dedicated to computer science and its history, entertainment software has a noteworthy role. In particular, young people may be involved in projects aimed to the development of toy games and mods, two kinds of software artefacts that can still be faced as one-person projects - that is, simple, personal, and rewarding.
\end{abstract}

Keywords: Computing History, Museum, Gamification, Game modding

\section{Introduction}

In 1985, the so-called Bodmer Report [1] set a pivotal point in relations among "science" and "public". There has never been a time when science and technology had been more related to progress and social wealth than in our age. Yet, people seem not to be really aware nor very interested in science. The Report pointed out such situation and set a new commitment in building the public understanding of science. Addressees of the Report were (and still are) schools, research communities, industries, political bodies, and, of course, museums dedicated to science and technology.

About thirty years after, the overall situation has not changed much. Still working to achieve the ultimate goal of public understanding, actors in the diffusion of scientific culture are today focused on strategic targets like public engagement. New ways have to be found to gain citizen interest and fascination, to involve them in the understanding and the development of science. Gamification is one strategy.

The paper presents the experiences of using gaming approaches carried out at the Museo degli Strumenti per il Calcolo (Museum of Computing Machinery, Museum in the following) [2] of the University of Pisa. As a Museum dedicated to computer science and its history, entertainment software plays a noteworthy role. 
Smartphones, tablets, videogame consoles are very popular. Yet, paradoxically, the public is not really interested in understanding the science behind them: it is satisfied by their simple usage, sometimes by their bare possession as status symbols.

With the initiatives concerning games and video games the Museum tries to raise the general interest in information technologies and gadgets up to engage the public, the youngers in particular, in "true" computer science.

\section{Games in the Museum experience}

Gamification is the usage of game dynamics to drive participation in non gaming contexts. While the term is recent, the idea is not. It is possible to find traces of planned gamification in the experiments of socialist competition like the Stakhanovite movement with all its surrounding mechanisms of records, awards and public praise [3]. The habit to gaming is however quite different today than in the days of Aleksei Stakhanov. For instance, thanks to video games there are much many gamers.

One of the recognized field of application for gamification is education [4] and Museums are committed in education. Moreover, they have to mark the difference with traditional teaching: museums are not schools and they have to attract and engage their public. No wonder that gamification is a sort of watchword.

In the following we will present three kinds of experiences carried out at the Museum. They can be seen as cases in gamification, but we prefer just to say that they concern games and computer games. For a rational and out-of-hype discussion about gamification in museums we suggest [5]. For a more comprehensive description of the Museum and of the underlying research project [6] we refer to [7].

\subsection{Retro-gaming}

Old video games have a characteristic vintage appeal for the large public: they played a large part in the childhood of many; even if they appear awkward and primitive to young people, they are still recognized as games. Many brands are still active. Temporary exhibits dedicated to video games are frequent events, organized by important institutions like the Cité des Sciences [8], or the Smithsonian [9]. There are also Museums dedicated to video games, one of the most known is in Berlin [10].

The Night of Old Video Games is a tournament that we organize as a yearly event, plus special editions. Qualifications and early phases are held in the afternoon and the final challenges in the evening. Due to the high appeal of old video games, the event is an easy success in terms of participation by players and spectators - a valuable mean of visibility for the Museum. But the initiative has also more noble goals.

As part of the schedule of the Night, brief speeches are held. They are used to talk about computer history topics. For instance, the last two editions were about Super Mario Bros. on the Nintendo Entertainment System, and Ghosts'n Goblins on the Commodore 64. The speeches covered topics with different perspectives:

1. Technology; like sound and graphics features of the NES and the $\mathrm{C}=64$;

2. Game art; Mario is a long-running and successful franchise; the musical score of Ghosts 'n Goblins was a recognised masterpiece; 
3. Economy and culture; Nintendo has celebrated 125 years of presence in the game industry, Commodore had a terrific success in the $80 \mathrm{~s}$, then is disappeared; both are good examples to discuss the ability to industrially exploit the opportunities offered by the available technologies.

During the tournament, the majority of the matches are played on software emulators running on common modern PCs; only the finals take place on the original consoles or computers. Playing on the originals has to be perceived as a special reward and a rare occasion. It is a way to educate to respect and preserve the relics of the past. Moreover, the emulators are a link to the present with a peculiar view on the evolution of computers: software can virtually make eternal the hardware.

\subsection{Challenges on Old Machines}

Some of the educational activities of the Museum challenge the visitors to operate old machines. Using an old, strange device is a game of ability with a number of difficulties and the natural consequent satisfaction when the task succeeds.

It is easy to engage people by let them play with an old mechanical calculator like a Brunsviga. The same happens for the hardware replica of the digital adder of the first Italian computer. We can let people operate the replica under supervision. The same is for pieces in the collection that are not very rare/antique and are sufficiently sturdy. The feeling of having the special opportunity to operate an uncommon old piece of computing machinery helps the visitor to enjoy the moment.

When the machines are very rare relics it is not possible to jeopardize them. Each usage wears the mechanisms a little more: it has to be permitted only in very special occasions and by expert hands only. In these cases we use software simulators.

Exploiting the success of "The Imitation Game" movie, in March the Museum organized an exhibition and a series of events played on the difference between the fiction and the real facts. We had an actual Enigma machine and we showed it working. To let the public experience the Enigma, we used simulators to re-enact the true World War II routines used to set-up the daily key and to encrypt messages. Being the procedures (the actual ones, not the babble you see in the movie) quite complicated and error prone, it was a real endeavour for visitors and students to successfully complete a whole cycle of set-up, encode and decode.
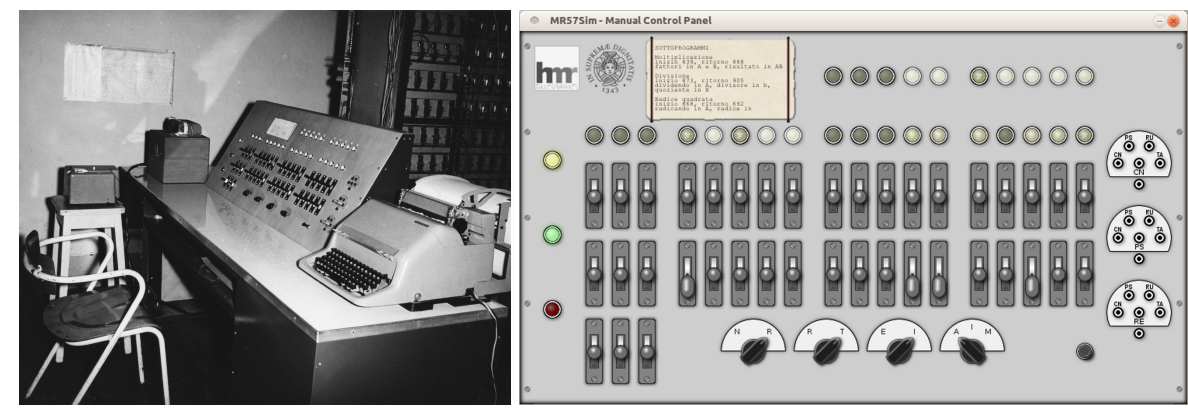

Fig. 1. A photo of the Macchina Ridotta and a screenshot of the simulator. 
The simulator of the Macchina Ridotta (MR), is one of the most relevant results of the HMR project [11]. The MR was dismantled in late 1958 to reuse its electronic components in a second computer: no relics of the very first Italian computer survived. The simulator was developed starting from the retrieved blueprints and technical reports: today it makes possible to see the MR running again [12]. With such a background, the MR simulator is a set piece of the educational activities of the $\mathrm{Mu}$ seum. It is used to show the principles and the basic mechanisms that make the computers work. The main difference between the old computers and their modern descendants is that the former expose all their inner workings while the latter hide them behind friendly interfaces. Of course, operating the MR is awkward for today standards (see Fig. 1): its user interface is made of many switches and lights used to input and output binary data.

The simulators are software pieces, but they are not video games. It is the aura of old (even lost) machines, the hurdle of the complex procedures, the oddity of the interfaces and the way the challenge is thrown that build up the gaming feeling.

\subsection{Ad hoc Edugames}

The Museum is also experimenting the development of its own edugames. The occasion was given by the theme that the Tuscany Region proposed in the call for the events organized for the Museum Night 2015: sustainability.

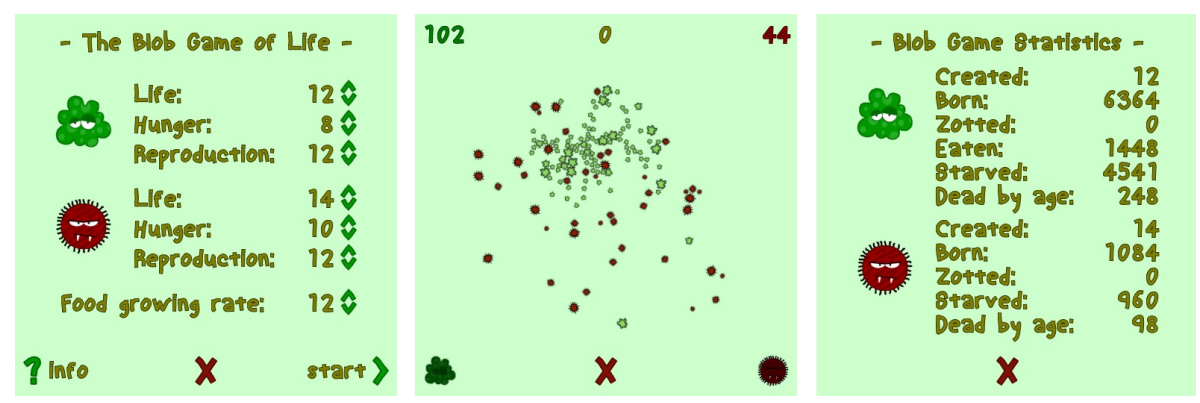

Fig. 2. Three screenshots of the prey-predator simulation game

We decided to use the Night to narrate the fragile equilibria of Nature, on which the sustainability of the Planet relies. Being a Museum devoted to computing, our interpretation of such idea is based on mathematical models and the ability to show them live using computer simulation technologies. For this purpose we developed a simple game to experiment with a small closed ecosystem where two species, prey and predators, interact. The game (see Fig. 2) lets the player modify the fundamental parameters of the species and observe the evolution of the ecosystem. Like a god, she/he may (or may not) intervene to create and kill individuals trying to recover a precarious equilibrium or to alter it to see what happens.

Besides ecosystems, the game is a good starting point to talk about historical mathematical models (like the Lotka-Volterra one) as well as modern simulation techniques (like the agent-based one) [13]. Moreover, the game is a case of software de- 
velopment taken on by the Museum itself, actually an one-man-project: a very small investment when compared to mainstream video game titles. We want to demonstrate to our public that programming may still be an activity on a human scale.

\section{Further Engagement with Toy Games and Mods}

Every museum can use video games to drive participation and engage its public. A Museum dedicated to computer science has a further motivation: video games are part of the history it has to preserve, study and disseminate. In this paper we presented the activities of our Museum which concern games and computer games.

Moreover, we believe that games can further help the building of public understanding of computer science. Games have a particular role in the history of computing. They were present since the beginning and were used in researches ranging from artificial intelligence up to human-machine interaction. When in the 80 s information technology became a mass phenomenon, video games and home computers fired up curiosity about computer science and popularized programming up to the status of a hobby. Many people learned programming and enjoyed it as a new kind of Meccano.

We propose to renew those days by engaging people, the youngsters in particular, on simple software development projects. We identified toy games and mods as perfectly sized kinds of projects. Toy games are little video games, built using rapid development environments, relying on $2 \mathrm{D}$ graphics and based more on game dynamics than on elaborate plots and gorgeous sceneries. Mods [14] are modifications made to existing video games to change or add contents like equipment items, characters, sceneries, behaviours. Toy games and mods are software artefacts that can still be faced as one-man projects - that is, simple, personal, and rewarding.

An experiment about toy games is already in progress. The first cycle of the Laboratorio di Videogiochi (Video Game Lab in Italian) has been held from November 2014 to June 2015. Based on a weekly schedule, the Lab offers a crash course in gaming history, architecture, technologies and development processes, followed by a substantial set of programming lessons using Stencyl [15] to develop a game. The course was followed by a small class of 12 students of which only 6 were able to completely fulfill the final assignments.

While it is not possible to derive definitive conclusion, we can say that is difficult to recreate the happy moment of the $80 \mathrm{~s}$. The effort required to develop a toy game is low, but not insignificant and sufficient to discourage many. As a consequence, a modding lab is seen as an interesting alternative to engage the public in game development and has therefore been considered for future implementation among the activities held at the Museum.

Meanwhile, we want to stress the need of support from the industry of video games and electronic gadgets. The PC platform has to be supported as well as the moddability of the video games: this is the precondition to let people try, with reasonable effort, to be part of the amazing world of video game development.

The situation about mobile platform is more complex. The popularity of these devices is a great opportunity to engage people. However, it is hindered by the rise of the walled garden model of app distribution. This model dictates that apps have to be 
thoroughly scrutinized by platform owners before they can be made available. In some cases the store requires a fee even if the app is free. This is a large setback from the days of home computing.

Developing games for mobile platforms is possible, but the need of an external programming environment and the complicated distribution policies surely does not encourage people to try to program and to discover true computer science.

\section{References}

1. Bodmer W.F. (ed.): The Public Understanding of Science. The Royal Society (1985)

2. Museo degli Strumenti per il Calcolo. http://www.facebook.com/MuseoStrumentiCalcolo (accessed July 3, 2015)

3. Nelson M.J.: Soviet and American precursors to the gamification of work. In: Proc. of the 16th International Academic MindTrek Conference, pp. 23-26. ACM (2012)

4. de Sousa Borges S. et alii: A systematic mapping on gamification applied to education. In: Proc. of the 29th Symposium on Applied Computing, pp. 216-222. ACM (2014)

5. Rodley E.: Gaming the museum - separating fad from function. http://exhibitdev.wordpress.com/2011/07/07/gaming-the-museum-separating-fad-from-function-part-four-of (accessed July 3, 2015)

6. Hackerando la Macchina Ridotta. http://hmr.di.unipi.it (accessed July 3, 2015)

7. Cignoni G.A., Gadducci F.: Using Old Computers for Teaching Computer Science. In: Tatnal A. et al. (eds.): Making the History of Computing Relevant - IFIP WG 9.7 International Conference, pp. 121-131. Springer (2013)

8. Jeu Vidéo. Cité des Sciences. http://www.cite-sciences.fr/fr/au-programme/expos-temporaires/jeuvideo-lexpo/accueil-de-la-cite-du-jeu-video (accessed July 3, 2015)

9. The Art of Videogames. Smithsonian American Art Museum. http://americanart.si.edu/exhibitions/archive/2012/games (accessed July 3, 2015)

10. Computerspielemuseum. http://www.computerspielemuseum.de (accessed July 3, 2015)

11. Cignoni G.A., Gadducci F.: Rediscovering the Very First Italian Digital Computer. In: Proceedings of 3rd IEEE History of Electro-technology Conference. IEEE (2012)

12. Cignoni G.A., Gadducci F., Paci S.: A Virtual Experience on the Very First Italian Computer. In: ACM Journal of Computing and Cultural Heritage, Vol. 7, No. 4, pp. 21:1-21:23 (2015)

13. Kim S., Hoffmann C., Ramachandran V.: Analyzing the Parameters of Prey-Predator Models for Simulation Games in Entertainment Computing. Proc. of the IFIP International Conference on Entertainment Computing ICEC 2010. Lecture Notes in Computer Science Vol. 6243, pp. 216-223. Springer-Verlag (2010)

14. Cignoni G.A.: Reporting on the Mod software process. In: Proc. of the VIII European Workshop on Software Process Technology, Lecture Notes in Computer Science, Vol. 2077, pp. 242-245. Springer-Verlag (2001)

15. Stencyl - Amazing Games without Code. http://www.stencyl.com (accessed July 3, 2015) 